\title{
CHARACTERISTIC CLASSES AND OBSTRUCTION THEORY FOR INFINITE LOOP SPACES
}

\author{
Dominique Arlettaz
}

The classical extension problem is to determine whether or not a given map $g: A \rightarrow Y$, defined on a given subspace $A$ of a space $X$, has an extension $X \rightarrow Y$. The present paper examines this question in the special case where the $k$-invariants of $Y$ are cohomology classes of finite order (for instance if $Y$ is an infinite loop space).

\section{Introduction}

Let $(X, A)$ be a relative $C W$-complex and $Y$ an $(m-1)$-connected simple $C W$-complex $(m \geq 1)$. The classical obstruction theory describes the primary obstruction $\gamma^{m+1}(g) \in H^{m+1}\left(X, A ; \pi_{m} Y\right)$ to extend a map $g: A \rightarrow Y$ to a map $X \rightarrow Y$, in term of the characteristic class $i^{m}(Y) \in H^{m}\left(Y ; \pi_{m} Y\right)$ as follows : $\gamma^{m+1}(g)=(-1)^{m} \delta g^{*}\left(i^{m}(Y)\right)$, where $g^{*}$ is the homomorphism induced by $g$ in cohomology and $\delta$ the coboundary operator of the cohomology sequence of the pair $(X, A)$. If $\gamma^{m+1}(g)=0$, then there is an extension of $g$ to the $(m+1)$ dimensional skeleton of $(X, A)$; but the vanishing of this primary obstruction is in general not sufficient in order to determine whether or not it is possible to extend the map $g$ to $X$, and one must consider higher obstructions, which have a more difficult description.

The purpose of this paper is to provide such a description for the case where $Y$ is a space with Postnikov $k$-invariants of finite order (for example an infinite loop space). For these spaces we define in Section $1 n$-dimensional characteristic classes $j^{n}(Y) \in H^{n}\left(Y ; \pi_{n} Y\right)$ for all positive integers $n$. Section 2 gives some examples of these characteristic classes, in connection with the cohomology of certain classical groups. Finally, Section 3 is devoted to the 


\section{ARLETTAZ}

extension problem : for all positive integers $n$ we define obstruction classes $\zeta^{n+1}(g) \in H^{n+1}\left(X, A ; \pi_{n} Y\right)$ which are related to the characteristic classes of $Y$ by the (similar) formula $\zeta^{n+1}(g)=(-1)^{n} \delta g^{*}\left(j^{n}(Y)\right)$, and we show that, under suitable conditions (for instance after localization of the target space $Y$ ), the extensibility of the map $g$ is equivalent to the vanishing of these classes.

I would like to thank Professor A. Dold for his valuable comments.

\section{Characteristic classes}

If $Y$ is an $(m-1)$-connected space (with $\pi_{1} Y$ abelian if $m=1$ ), its characteristic class

$$
i^{m}(Y) \in H^{m}\left(Y ; \pi_{m} Y\right)
$$

is classically defined to be the element of $H^{m}\left(Y ; \pi_{m} Y\right)$ corresponding to the inverse of the Hurewicz isomorphism $\pi_{m} Y \stackrel{\cong}{\longrightarrow} H_{m}(Y ; \mathbb{Z})$ under the isomorphism $H^{m}\left(Y ; \pi_{m} Y\right) \cong H o m\left(H_{m}(Y ; \mathbb{Z}), \pi_{m} Y\right)$ given by the universal coefficient theorem (cf. [8, p.236]). The class $i^{m}$ is natural in the following sense : if $h: Y \rightarrow Y^{\prime}$ is a map between two $(m-1)$-connected spaces, then $h_{*}\left(i^{m}(Y)\right)=h^{*}\left(i^{m}\left(Y^{\prime}\right)\right)$, where the homomorphisms $h_{*}: H^{m}\left(Y ; \pi_{m} Y\right) \rightarrow H^{m}\left(Y ; \pi_{m} Y^{\prime}\right)$ and $h^{*}: H^{m}\left(Y^{\prime} ; \pi_{m} Y^{\prime}\right) \rightarrow H^{m}\left(Y ; \pi_{m} Y^{\prime}\right)$ are induced by $h$. Our objective is to define, for certain spaces, characteristic classes in all dimensions.

Let us start by explaining our notation. All spaces we consider in this section are connected simple $C W$-complexes. For such a space $Y$ and for any positive integer $n$, let $\alpha_{n}: Y \rightarrow Y[n]$ denote the $n$-th Postnikov section of $Y$ (i.e., $Y[n]$ is a $C W$-complex obtained form $Y$ by adjoining cells of dimension $\geq n+2$, with $\pi_{i} Y[n]=0$ for $i>n$ and $\left(\alpha_{n}\right)_{*}: \pi_{i} Y \stackrel{\cong}{\longrightarrow} \pi_{i} Y[n]$ for $i \leq n$ ), and $k^{n+1}(Y)$ the Postnikov $k$-invariant in $H^{n+1}\left(Y[n-1] ; \pi_{n} Y\right): k^{n+1}(Y)$ is a homotopy class of maps $Y[n-1] \rightarrow K\left(\pi_{n} Y, n+1\right)$ such that, if $K\left(\pi_{n} Y, n\right) \rightarrow$ $P K\left(\pi_{n} Y, n+1\right) \stackrel{p}{\longrightarrow} K\left(\pi_{n} Y, n+1\right)$ is the path-fibration over $K\left(\pi_{n} Y, n+1\right)$ and if $W_{n}$ is the pull-back of $\left(k^{n+1}(Y), p\right)$, there exists a (non-unique) homotopy equivalence $\theta: Y[n] \stackrel{\simeq}{\longrightarrow} W_{n}$.

In order to introduce the notion of $n$-dimensional characteristic classes for a space $Y$, we must assume that $n$ is a positive integer such that the $k$-invariant $k^{n+1}(Y)$ is an element of finite order, say $s_{n}^{Y}$, in the group $H^{n+1}\left(Y[n-1] ; \pi_{n} Y\right)$ 


\section{ARLETTAZ}

(notice that $k^{n+1}(Y)$ is trivial for all $n \leq m$ if $Y$ is $(m-1)$-connected). For instance, this condition is satisfied for all $n \geq 1$ if $Y$ is an $H$-space of finite type (cf. [1, Proposition 4.1]). Other examples are given in [3] where the following theorem is proved : there exist positive integers $S_{t}(t \in \mathbb{Z})$ such that $S_{n-m+1} k^{n+1}(Y)=0$ for $n \leq r+2 m-2$ if $Y$ is an $(m-1)$-connected $r$-fold loop space ( $m \geq 1, r \geq 0)$; in particular, all $k$-invariants of an infinite loop space have finite order.

Under this hypothesis, it is possible to construct a map

$$
f_{n}^{Y}: Y \rightarrow K\left(\pi_{n} Y, n\right)
$$

which induces multiplication by $s_{n}^{Y}$ on $\pi_{n} Y$ (cf. [2, Lemma 4]). Look at the commutative diagram

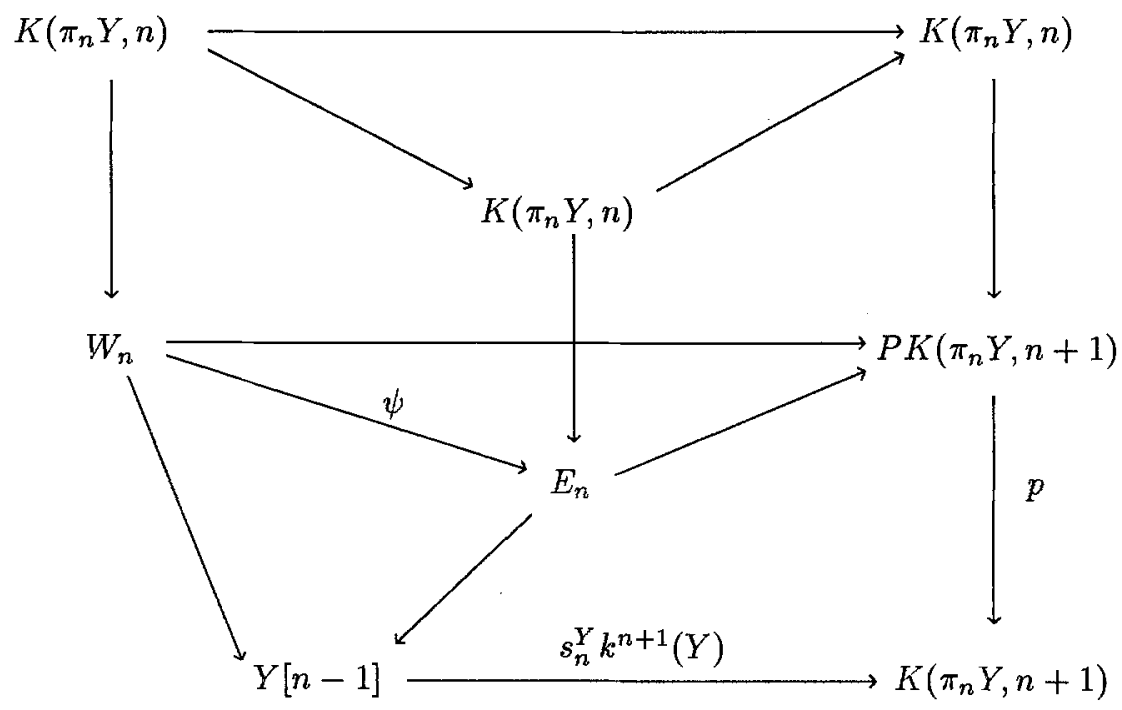

where each column is a fibration and $E_{n}$ the pull-back of $\left(s_{n}^{Y} k^{n+1}(Y), p\right)$; this implies the existence of the map $\psi$, and the fact that $s_{n}^{Y} k^{n+1}(Y)$ is homotopic to the constant map produces a homotopy equivalence $E_{n} \simeq Y[n-1] \times K\left(\pi_{n} Y, n\right)$. 


\section{ARLETTAZ}

Observe that $\psi$ induces multiplication by $s_{n}^{Y}$ on $\pi_{n} Y$ since $s_{n}^{Y} k^{n+1}(Y)$ is actually the composition of $k^{n+1}(Y)$ with $s_{n}^{Y}$. identity: $K\left(\pi_{n} Y, n+1\right) \rightarrow$ $K\left(\pi_{n} Y, n+1\right)$. We write $f_{n}^{Y}$ for the composition

$$
Y \stackrel{\alpha_{n}}{\rightarrow} Y[n] \stackrel{\theta}{\simeq} W_{n} \stackrel{\psi}{\rightarrow} E_{n} \simeq Y[n-1] \times K\left(\pi_{n} Y, n\right) \stackrel{\pi}{\rightarrow} K\left(\pi_{n} Y, n\right) \stackrel{\eta}{\simeq} K\left(\pi_{n} Y, n\right)
$$

where $\pi$ denotes the projection onto the second factor and $\eta$ a map inducing an isomorphism on $\pi_{n} Y$ such that the induced homomorphism $\left(f_{n}^{Y}\right)_{*}: \pi_{n} Y \rightarrow \pi_{n} Y$ is exactly multiplication by $s_{n}^{Y}$. This map $f_{n}^{Y}$ is not unique.

Definition 1.1. If $Y$ is a connected simple $C W$-complex and $n$ a positive integer such that $k^{n+1}(Y)$ is a cohomology class of finite order $s_{n}^{Y}$, an $n$-dimensional characteristic map for $Y$ is a map

$$
f_{n}^{Y}: Y \longrightarrow K\left(\pi_{n} Y, n\right)
$$

which induces mutliplication by $s_{n}^{Y}$ on $\pi_{n} Y$. The $n$-dimensional characteristic class of $Y$ associated with $f_{n}^{Y}$ is

$$
j^{n}(Y):=\left(f_{n}^{Y}\right)^{*}\left(i^{n}\left(K\left(\pi_{n} Y, n\right)\right)\right) \in H^{n}\left(Y ; \pi_{n} Y\right)
$$

where $\left(f_{n}^{Y}\right)^{*}$ is the homomorphism induced by $f_{n}^{Y}$ in cohomology (in other words, $j^{n}(Y)$ is the cohomology class corresponding to the homotopy class of $\left.f_{n}^{Y}\right)$.

The characteristic class $j^{n}(Y)$ is not uniquely defined since it depends on the map $f_{n}^{Y}$. The fibre of the Postnikov section $Y\left[n \mid \rightarrow Y[n-1]\right.$ is $K\left(\pi_{n} Y, n\right)$ and we call $\rho$ the inclusion map $K\left(\pi_{n} Y, n\right) \hookrightarrow Y[n]$; because of the isomorphism $\left(\alpha_{n}\right)^{*}: H^{n}\left(Y[n] ; \pi_{n} Y\right) \stackrel{\cong}{\longrightarrow} H^{n}\left(Y ; \pi_{n} Y\right)$, we may consider the induced homomorphism $\rho^{*}: H^{n}\left(Y ; \pi_{n} Y\right) \rightarrow H^{n}\left(K\left(\pi_{n} Y, n\right) ; \pi_{n} Y\right)$. It is then obvious that all $n$-dimensional characteristic classes $j^{n}(Y)$ of $Y$ have the same image under $\rho$. In fact, we write $\rho$ for the composition of this map with a self-equivalence of $K\left(\pi_{n} Y, n\right)$, such that

$$
\left.\rho^{*}\left(j^{n}(Y)\right)=s_{n}^{Y} i^{n} K\left(\pi_{n} Y, n\right)\right)
$$




\section{ARLETTAZ}

Definition 1.2. $J^{n}(Y)$ is the image of any $n$-dimensional characteristic class $j^{n}(Y)$ of $Y$ under the homomorphism $H^{n}\left(Y ; \pi_{n} Y\right) \rightarrow H^{n}\left(Y ; \pi_{n} Y\right) / \operatorname{Ker} \rho^{*}$. $J^{n}(Y)$ is uniquely determined.

The remainder of this section establishes some elementary properties of these classes.

Proposition 1.3. If $Y$ is an $(m-1)$-connected simple $C W$-complex, then $j^{m}(Y)$ is uniquely determined and $i^{m}(Y)=j^{m}(Y)=J^{m}(Y)$.

Proof. If $Y$ is $(m-1)$-connected, $k^{m+1}(Y)$ is trivial $\left(s_{m}^{Y}=1\right)$ and $Y[m]=$ $K\left(\pi_{m} Y, m\right)$; thus, $\rho^{*}$ is an isomorphism, $j^{m}(Y)$ is unique and $j^{m}(Y)=J^{m}(Y)$. Since any $m$-dimensional characteristic map $f_{m}^{Y}: Y \rightarrow K\left(\pi_{m} Y, m\right)$ induces identity on $\pi_{m} Y$, the naturality of $i^{m}$ implies :

$$
j^{m}(Y)=\left(f_{m}^{Y}\right)^{*}\left(i^{m}\left(K\left(\pi_{m} Y, m\right)\right)\right)=i^{m}(Y)
$$

Let us discuss the naturality of $J^{n}$.

Proposition 1.4. Let $Y$ and $Y^{\prime}$ be connected simple $C W$-complexes, $n$ a positive integer such that $k^{n+1}(Y)$ and $k^{n+1}\left(Y^{\prime}\right)$ have finite order $s_{n}^{Y}$ and $s_{n}^{Y^{\prime}}$ respectively, $h: Y \rightarrow Y^{\prime}$ a map, and

$$
\begin{aligned}
& h_{*}: H^{n}\left(Y ; \pi_{n} Y\right) / \operatorname{Ker} \rho^{*} \rightarrow H^{n}\left(Y ; \pi_{n} Y^{\prime}\right) / \operatorname{Ker} \rho^{*} \text { and } \\
& h^{*}: H^{n}\left(Y^{\prime} ; \pi_{n} Y^{\prime}\right) / \operatorname{Ker} \rho^{*} \rightarrow H^{n}\left(Y ; \pi_{n} Y^{\prime}\right) / \operatorname{Ker} \rho^{*}
\end{aligned}
$$

the homomorphisms induced by $h$. Then

$$
s_{n}^{Y^{\prime}} h_{*}\left(J^{n}(Y)\right)=s_{n}^{Y} h^{*}\left(J^{n}\left(Y^{\prime}\right)\right)
$$

Proof. Look at the commutative diagram

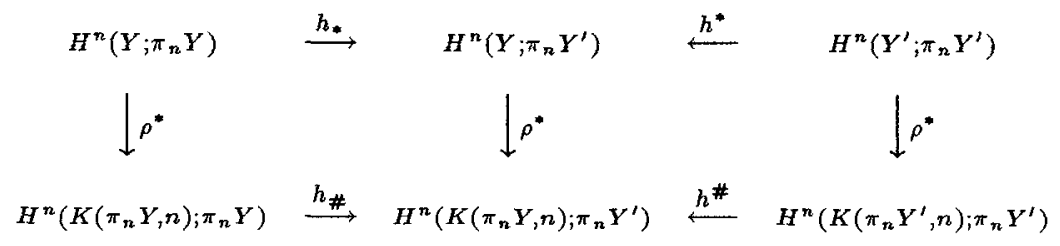


where the homomorphisms $h_{*}, h^{*}, h_{\#}, h^{\#}$ are induced by $h$. It follows from $h_{\#}\left(i^{n}\left(K\left(\pi_{n} Y, n\right)\right)\right)=h^{\#}\left(i^{n}\left(K\left(\pi_{n} Y^{\prime}, n\right)\right)\right)$ that $\rho^{*}\left(s_{n}^{Y^{\prime}} h_{*}\left(j^{n}(Y)\right)\right)=$ $s_{n}^{Y^{\prime}} h_{\#} \rho^{*}\left(j^{n}(Y)\right)=s_{n}^{Y^{\prime}} h_{\#}\left(s_{n}^{Y}\left(i^{n}\left(K\left(\pi_{n} Y, n\right)\right)\right)\right)=s_{n}^{Y} h^{\#}\left(s_{n}^{Y^{\prime}}\left(i^{n}\left(K\left(\pi_{n} Y^{\prime}, n\right)\right)\right)\right)$ $=s_{n}^{Y} h^{\#} \rho^{*}\left(j^{n}\left(Y^{\prime}\right)\right)=\rho^{*}\left(s_{n}^{Y} h^{*}\left(j^{n}\left(Y^{\prime}\right)\right)\right)$ for all $j^{n}(Y)$ and $j^{n}\left(Y^{\prime}\right)$. This becomes $s_{n}^{Y^{\prime}} h_{*}\left(J^{n}(Y)\right)=s_{n}^{Y} h^{*}\left(J^{n}\left(Y^{\prime}\right)\right)$ in the quotient $H^{n}\left(Y ; \pi_{n} Y^{\prime}\right) / \operatorname{Ker} \rho^{*}$.

Remark 1.5. Let $Y$ be a connected simple $C W$-complex, $n$ a positive integer with $k^{n+1}(Y)$ of finite order $s_{n}^{Y}, R$ a subring of the field of rationals $\mathbb{Q}$ such that $s_{n}^{Y}$ is invertible in $R, \ell: Y \rightarrow Y_{R}$ the localization map, and $\ell_{*}: H^{n}\left(Y ; \pi_{n} Y\right) \rightarrow H^{n}\left(Y ; \pi_{n} Y \otimes R\right)$ and $\ell^{*}: H^{n}\left(Y_{R} ; \pi_{n} Y \otimes R\right) \rightarrow H^{n}\left(Y ; \pi_{n} Y \otimes\right.$ $R$ ) the homomorphisms induced by $\ell$. The behaviour of $J^{n}$ under the localization map is described by the previous proposition : since $\ell$ localizes the $k$-invariants (cf. [7, Theorem 2.3]), $k^{n+1}\left(Y_{R}\right)$ is trivial and $\ell_{*}\left(J^{n}(Y)\right)=$ $s_{n}^{Y} \ell^{*}\left(J^{n}\left(Y_{R}\right)\right)$.

The same is actually true for $j^{n}:$ if $j^{n}(Y) \in H^{n}\left(Y ; \pi_{n} Y\right)$ is an $n$ dimensional characteristic class of $Y$, there exists an $n$-dimensional characteristic class $j^{n}\left(Y_{R}\right) \in H^{n}\left(Y_{R} ; \pi_{n} Y \otimes R\right)$ satisfying the relation

$$
\ell_{*}\left(j^{n}(Y)\right)=s_{n}^{Y} \ell^{*}\left(j^{n}\left(Y_{R}\right)\right)
$$

In order to prove this, consider an $n$-dimensional characteristic map $f_{n}^{Y}$ : $Y \rightarrow K\left(\pi_{n} Y, n\right)$ corresponding to $j^{n}(Y)$ and call $f_{n}^{Y} \otimes R: Y_{R} \rightarrow K\left(\pi_{n} Y \otimes R, n\right)$ its localization. The composition of $f_{n}^{Y} \otimes R$ with a map $K\left(\pi_{n} Y \otimes R, n\right) \rightarrow$ $K\left(\pi_{n} Y \otimes R, n\right)$ inducing multiplication by $1 / s_{n}^{Y}$ on $\pi_{n} Y \otimes R$ is an $n$-dimensional characteristic map for $Y_{R}$. Therefore, the induced homomorphism $\left(f_{n}^{Y} \otimes R\right)^{*}$ : $H^{n}\left(K\left(\pi_{n} Y \otimes R, n\right) ; \pi_{n} Y \otimes R\right) \rightarrow H^{n}\left(Y_{R} ; \pi_{n} Y \otimes R\right) \operatorname{maps} i^{n}\left(K\left(\pi_{n} Y \otimes R, n\right)\right)$ onto $s_{n}^{Y} j^{n}\left(Y_{R}\right)$ for some $n$-dimensional characteristic class $j^{n}\left(Y_{R}\right)$ of $Y_{R}$. The commutative diagram

$$
\begin{aligned}
& H^{n}\left(K\left(\pi_{n} Y, n\right) ; \pi_{n} Y\right) \stackrel{\ell_{\#}}{\longrightarrow} H^{n}\left(K\left(\pi_{n} Y, n\right) ; \pi_{n} Y \otimes R\right) \stackrel{\ell^{\#}}{\longleftarrow} H^{n}\left(K\left(\pi_{n} Y \otimes R, n\right) ; \pi_{n} Y \otimes R\right) \\
& \downarrow\left(f_{n}^{Y}\right)^{*} \quad \downarrow\left(f_{n}^{Y}\right)^{*} \quad \downarrow\left(f_{n}^{Y} \otimes R\right)^{*} \\
& H^{n}\left(Y ; \pi_{n} Y\right) \quad \stackrel{\ell_{*}}{\longrightarrow} \quad H^{n}\left(Y ; \pi_{n} Y \otimes R\right) \quad \stackrel{\ell^{*}}{\longleftarrow} \quad H^{n}\left(Y_{R} ; \pi_{n} Y \otimes R\right)
\end{aligned}
$$

completes the argument $: \ell_{*}\left(j^{n}(Y)\right)=\left(f_{n}^{Y}\right)^{*} \ell_{\#}\left(i^{n}\left(K\left(\pi_{n} Y, n\right)\right)\right)=$ 


\section{ARLETTAZ}

$\left(f_{n}^{Y}\right)^{*} \ell^{\#}\left(i^{n}\left(K\left(\pi_{n} Y \otimes R, n\right)\right)\right)=\ell^{*}\left(s_{n}^{Y} j^{n}\left(Y_{R}\right)\right)$.

We determine finally the relationship between the characteristic classes of a space $Y$ and those of its loop space $\Omega Y$. The cohomology suspension $\sigma^{*}$ induces the commutative diagram

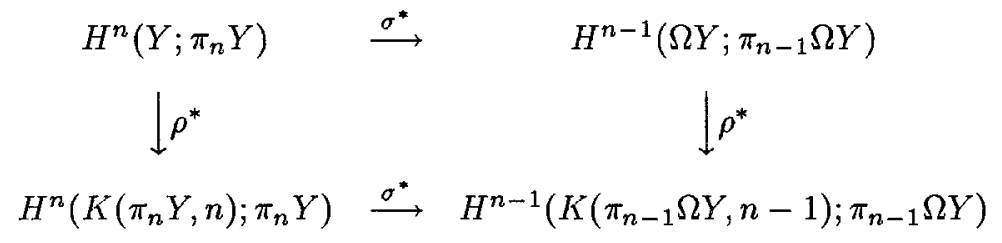

and a homomorphism

$$
\sigma^{*}: H^{n}\left(Y ; \pi_{n} Y\right) / \operatorname{Ker} \rho^{*} \rightarrow H^{n-1}\left(\Omega Y ; \pi_{n-1} \Omega Y\right) / \operatorname{Ker} \rho^{*}
$$

Proposition 1.6. Let $Y$ be a connected simple $C W$-complex and $n$ a positive integer such that $k^{n+1}(Y)$ has finite order $s_{n}^{Y}$. Then

$$
\sigma^{*}\left(J^{n}(Y)\right)=\left(s_{n}^{Y} / s_{n-1}^{\Omega Y}\right) J^{n-1}(\Omega Y)
$$

where $s_{n-1}^{\Omega Y}$ is the order of $k^{n}(\Omega Y)$.

Proof. Since $k^{n}(\Omega Y)$ is the image of $k^{n+1}(Y)$ under the cohomology suspension (cf. [8, p. 438]), it has finite order $s_{n-1}^{\Omega Y}$ dividing $s_{n}^{Y}$. Let $j^{n}(Y) \in$ $H^{n}\left(Y ; \pi_{n} Y\right)$ be an $n$-dimensional characteristic class of $Y$. It follows from $\rho^{*}\left(j^{n}(Y)\right)=s_{n}^{Y} i^{n}\left(K\left(\pi_{n} Y, n\right)\right)$ that $\rho^{*} \sigma^{*}\left(j^{n}(Y)\right)=\sigma^{*}\left(s_{n}^{Y} i^{n}\left(K\left(\pi_{n} Y, n\right)\right)\right)=$ $s_{n}^{Y} i^{n-1}\left(K\left(\pi_{n-1} \Omega Y, n-1\right)\right)=\left(s_{n}^{Y} / s_{n-1}^{\Omega Y}\right) \rho^{*}\left(j^{n-1}(\Omega Y)\right)$ for any $(n-1)$-dimensional characteristic class of $\Omega Y$. Therefore we get $: \sigma^{*}\left(J^{n}(Y)\right)=$ $\left(s_{n}^{Y} / s_{n-1}^{\Omega Y}\right) J^{n-1}(\Omega Y)$.

\section{Examples.}

(a) Consider the infinite loop space $B U$. There exist characteristic classes $j^{n}(B U) \in H^{n}\left(B U ; \pi_{n} B U\right)$ for all positive integers $n$, but if $n$ is odd, the vanishing of $\pi_{n} B U$ implies clearly that $j^{n}(B U)=0$; more interesting are the even dimensions since $\pi_{2 t} B U \cong \mathbb{Z}$ for any $t \geq 1$. As usual let us call $c_{t}$ the $t$-th universal Chern class in $H^{2 t}(B U ; \mathbb{Z})$. 
Proposition 2.1. Let $t$ be a positive integer. If $j^{2 t}(B U)$ is any $2 t$ dimensional characteristic class of $B U$, then $j^{2 t}(B U)= \pm c_{t}+$ decomposable elements.

Proof. It is known that $k^{2 t+1}(B U)$ has order $(t-1) !$ (cf. [6, Lemma 4.4]). Thus, if $\rho^{*}: H^{2 t}(B U ; \mathbb{Z}) \rightarrow H^{2 t}(K(\mathbb{Z}, 2 t) ; \mathbb{Z})$ is the homomorphism defined in Section $1, \rho^{*}\left(j^{2 t}(B U)\right)=(t-1) ! i^{2 t}(K(\mathbb{Z}, 2 t))$. On the other hand it is also proved in [6, Lemma 4.5] that $\rho^{*}\left(c_{t}\right)= \pm(t-1) ! i^{2 t}(K(\mathbb{Z}, 2 t))$. Consequently, $j^{2 t} \mp c_{t}$ belongs to the kernel of $\rho^{*}$, which is generated by products of the Chern classes $c_{1}, c_{2}, \ldots, c_{t-1}$.

(b) Let $A$ be the field of rationals $\mathbb{Q}$ or the ring of integers $\mathbb{Z}, S L(A)$ its infinite special linear group, and $Y:=B S L(A)^{+}$the simply connected space obtained by performing the plus construction on the classifying space of $S L(A)$. It is known by Borel's computation [4] that the rational cohomology of $Y$ is an exterior algebra generated by elements of degree $4 t+1, t \geq 1$ :

$$
H^{*}\left(B S L(A)^{+} ; \mathbb{Q}\right)=\Lambda\left(x_{5}, x_{9}, \ldots, x_{4 t+1}, \ldots\right) .
$$

Since $Y$ is an infinite loop space, we may consider characteristic classes $j^{n}(Y) \epsilon$ $H^{n}\left(Y ; \pi_{n} Y\right)$ for all $n \geq 1$. We want to show that the classes provide a description of the generators $x_{4 t+1}, t \geq 1$. Consider the localization map $\ell: Y \rightarrow Y_{\mathbb{Q}}$ (i.e., the rational type of $Y$ ). According to [4], $\pi_{n} Y_{\mathbb{Q}} \cong \mathbb{Q}$ if $n \equiv 1 \bmod 4$, $n \geq 5$, and $\pi_{n} Y_{\mathbb{Q}}=0$ otherwise. Therefore, the map $\ell$ induces the homomorphism $\ell_{*}: H^{4 t+1}\left(Y ; \pi_{4 t+1} Y\right) \rightarrow H^{4 t+1}(Y ; Q)$ for $t \geq 1$.

Proposition 2.2. For $t \geq 1$, it is possible to choose

$$
x_{4 t+1}=\ell_{*}\left(j^{4 t+1}\left(B S L\left(A^{+}\right)\right) .\right.
$$

Proof. For any $t \geq 1$, let $j^{4 t+1}(Y)$ be a $(4 t+1)$-dimensional charactersitic class of $Y$ and $j^{4 t+1}\left(Y_{\mathbb{Q}}\right)$ the corresponding characteristic class of $Y_{\mathbb{Q}}$ given by Remark 1.5. Since the $k$-invariants $k^{n+1}(Y)$ have finite order $s_{n}^{Y}$ for all $n \geq 1, Y_{\mathbb{Q}}$ is a product of Eilenberg-MacLane spaces : $Y_{\mathbb{Q}}=\prod_{t=1}^{\infty} K(\mathbb{Q}, 4 t+1)$. Its rational cohomology is then an exterior algebra generated by the classes $j^{4 t+1}\left(Y_{\mathbb{Q}}\right), t \geq 1$. Using the isomorphism $\ell^{*}: H^{*}\left(Y_{\mathbb{Q}} ; \mathbb{Q}\right) \stackrel{\cong}{\rightrightarrows} H^{*}(Y ; \mathbb{Q})$, we 


\section{ARLETTAZ}

may choose $x_{4 t+1}=s_{4 t+1}^{Y} \ell^{*}\left(j^{4 t+1}\left(Y_{\mathbb{Q}}\right)\right)$ and deduce from Remark 1.5. that $x_{4 t+1}=\ell_{*}\left(j^{4 t+1}(Y)\right)$ for $t \geq 1$.

Notice that $\ell_{*}\left(j^{n}\left(B S L(A)^{+}\right)\right)=0$ if $n \not \equiv 1 \bmod 4$.

The same argument produces analogous assertions for the generators of the rational cohomology of $S p(A)$ and $O(A)(\mathrm{cf} .[4])$ :

$$
\begin{aligned}
& H^{*}\left(B S p(A)^{+} ; \mathbb{Q}\right)=\mathbb{Q}\left[y_{2}, y_{6}, \ldots, y_{4 t-2}, \ldots\right], \\
& H^{*}\left(B O(A)^{+} ; \mathbb{Q}\right)=\mathbb{Q}\left[z_{4}, z_{8}, \ldots, z_{4 t}, \ldots\right] .
\end{aligned}
$$

Proposition 2.3. For $t \geq 1$, it is possible to choose

$$
y_{4 t-2}=\ell_{*}\left(j^{4 t-2}\left(B S p(A)^{+}\right)\right) \text {and } z_{4 t}=\ell_{*}\left(j^{4 t}\left(B O(A)^{+}\right)\right) \text {. }
$$

Remark finally that similar results are obtained when $A$ is an imaginary quadratic number field or its ring of integers.

\section{Obstruction theory.}

The classical obstruction theory (cf. [5] or [8, $\S \mathrm{V} .5-6]$ ) examines the following problem : let $(X, A)$ be a relative $C W$-complex, $Y$ a connected simple $C W$-complex and $g$ a map $A \rightarrow Y$; the question is to determine whether or not $g$ can be extended over $X$.

If $Y$ is $(m-1)$-connected ( $m \geq 1)$, it is possible to extend $g$ over $X_{m}$, the $m$ dimensional skeleton of $(X, A)$. If $\bar{g}: X_{m} \rightarrow Y$ is such an extension, one defines a cocycle $c^{m+1}(\bar{g}) \in H^{m+1}\left(X_{m+1}, X_{m} ; \pi_{m} Y\right)$ whose vanishing corresponds to the extensibility of $\bar{g}$ over $X_{m+1}$, and one shows that if $\bar{g}$ and $\tilde{g}: X_{m} \rightarrow Y$ are extensions of $g$, then $c^{m+1}(\bar{g}) \sim c^{m+1}(\tilde{g})$ : consequently, there is a uniquely defined element

$$
\gamma^{m+1}(g) \in H^{m+1}\left(X, A ; \pi_{m} Y\right)
$$

which is the cohomology class of $c^{m+1}(\bar{g})$ for any extension $\bar{g}: X_{m} \rightarrow Y$ of $g$; $\gamma^{m+1}(g)$ is called the primary obstruction to extending $g$. It is related to the characteristic class $i^{m}(Y) \in H^{m}\left(Y ; \pi_{m} Y\right)$ of the target space $Y$ by the formula

$$
\gamma^{m+1}(g)=(-1)^{m} \delta g^{*}\left(i^{m}(Y)\right)
$$




\section{ARLETTAZ}

where $g^{*}$ denotes the homomorphism $H^{*}(Y ;-) \rightarrow H^{*}(A ;-)$ induced by $g$, and $\delta: H^{*}(A ;-) \rightarrow H^{*+1}(X, A ;-)$ the coboundary operator of the cohomology sequence of the pair $(X, A)$. The primary obstruction $\gamma^{m+1}(g)$ gives a partial solution to the extension problem : $\gamma^{m+1}(g)$ is trivial if and only if $g$ can be extended over $X_{m+1}$. But in general, there exist higher obstructions to extending $g$ over $X$, and it is hard to describe them.

The purpose of this section is to consider the extension problem in the following special situation: we assume that $g: A \rightarrow Y$ has an extension $\bar{g}: X_{n} \rightarrow Y$ and that the $k$-invariant $k^{n+1}(Y)$ of $Y$ has finite order $s_{n}^{Y}$ (but we do not assume that $Y$ is $(n-1)$-connected). The basic idea is to apply the classical theory to the composition of $g$ (respectively $\bar{g}$ ) with any $n$-dimensional characteristic map $f_{n}^{Y}: Y \rightarrow K\left(\pi_{n} Y, n\right)$ introduced in Section 1 .

Lemma 3.1. $c^{n+1}\left(f_{n}^{Y} \circ \bar{g}\right)=s_{n}^{Y} c^{n+1}(\bar{g}) \in H^{n+1}\left(X_{n+1}, X_{n} ; \pi_{n} Y\right)$.

Proof. An elementary property of the cocycle $c^{n+1}$ is that $c^{n+1}\left(f_{n}^{Y} \circ \bar{g}\right)=$ $\left(f_{n}^{Y}\right)_{*}\left(c^{n+1}(\bar{g})\right)$, where $\left(f_{n}^{Y}\right)_{*}: H^{n+1}\left(X_{n+1}, X_{n} ; \pi_{n} Y\right) \rightarrow H^{n+1}\left(X_{n+1}, X_{n}\right.$; $\left.\pi_{n} K\left(\pi_{n} Y, n\right)\right)$ is induced by $f_{n}^{Y}$. But, by definition, $\left(f_{n}^{Y}\right)_{*}$ is multiplication by $s_{n}^{Y}: c^{n+1}\left(f_{n}^{Y} \circ \bar{g}\right)=s_{n}^{Y} c^{n+1}(\bar{g})$.

It follows from this lemma that $c^{n+1}\left(f_{n}^{Y} \circ \bar{g}\right)$ does not depend on the choice of $f_{n}^{Y}$. If $\bar{g}$ and $\tilde{g}: X_{n} \rightarrow Y$ are extensions of $g$, then $c^{n+1}\left(f_{n}^{Y} \circ \bar{g}\right) \sim c^{n+1}\left(f_{n}^{Y} \circ \tilde{g}\right)$ since $K\left(\pi_{n} Y, n\right)$ is $(n-1)$-connected. Thus, we may give the following

Definition 3.2. $\zeta^{n+1}(g) \in H^{n+1}\left(X, A ; \pi_{n} Y\right)$ is the cohomology class of $s_{n}^{Y} c^{n+1}(\bar{g})$ for any extension $\bar{g}: X_{n} \rightarrow Y$ of $g: A \rightarrow Y$. It turns out that $\zeta^{n+1}(g)=\gamma^{n+1}\left(f_{n}^{Y} \circ g\right)$ for any $n$-dimensional characteristic map $f_{n}^{Y}$. Observe that this obstruction class $\zeta^{n+1}(g)$ is well defined, although $f_{n}^{Y}$ (respectively $\left.j^{n}(Y)\right)$ is not uniquely determined.

Proposition 3.3. $\zeta^{n+1}(g)=(-1)^{n} \delta g^{*}\left(j^{n}(Y)\right) \in H^{n+1}\left(X, A ; \pi_{n} Y\right)$ for any $n$-dimensional characteristic class $j^{n}(Y) \in H^{n}\left(Y ; \pi_{n} Y\right)$.

Proof. If $f_{n}^{Y}$ is an $n$-dimensional characteristic map corresponding to $j^{n}(Y)$, then $\zeta^{n+1}(g)=\gamma^{n+1}\left(f_{n}^{Y} \circ g\right)=(-1)^{n} \delta g^{*}\left(f_{n}^{Y}\right)^{*}\left(i^{n}\left(K\left(\pi_{n} Y, n\right)\right)\right)=$ $(-1)^{n} \delta g^{*}\left(j^{n}(Y)\right)$. 


\section{ARLETTAZ}

Remark 3.4. The obstruction class $\zeta^{n+1}$ has the following properties.

a) If $g_{0}, g_{1}: A \rightarrow Y$ are homotopic maps, extensible over $X_{n}$, then $\zeta^{n+1}\left(g_{0}\right)=\zeta^{n+1}\left(g_{1}\right)$.

b) Let $Y^{\prime}$ be another connected simple space with $k^{n+1}\left(Y^{\prime}\right)$ of finite or$\operatorname{der} s_{n}^{Y^{\prime}}, h$ a map $Y \rightarrow Y^{\prime}$ and $h_{*}: H^{n+1}\left(X, A ; \pi_{n} Y\right) \rightarrow H^{n+1}\left(X, A ; \pi_{n} Y^{\prime}\right)$ the homomorphism induced by $h$. Then $s_{n}^{Y} \zeta^{n+1}(h \circ g)=s_{n}^{Y^{\prime}} h_{*}\left(\zeta^{n+1}(g)\right) \in$ $H^{n+1}\left(X, A ; \pi_{n} Y^{\prime}\right)$ because both terms are equal to the cohomology class of $s_{n}^{Y} s_{n}^{Y^{\prime}} c^{n+1}(h \circ \bar{g})=s_{n}^{Y} s_{n}^{Y^{\prime}} h_{*}\left(c^{n+1}(\bar{g})\right)$.

c) If $h^{\prime}$ is a cellular map $\left(X^{\prime}, A^{\prime}\right) \rightarrow(X, A)$, then $\zeta^{n+1}\left(\left.g \circ h^{\prime}\right|_{A^{\prime}}\right)=$ $\left(h^{\prime}\right)^{*}\left(\zeta^{n+1}(g)\right) \in H^{n+1}\left(X^{\prime}, A^{\prime} ; \pi_{n} Y\right)$, where $\left(h^{\prime}\right)^{*}$ is the homomorphism induced by $h^{\prime}$ in cohomology.

Our objective is now to exhibit the relationships between the obstruction classes $\zeta^{n+1}(g)$ and the solution of the extension problem.

Theorem 3.5. Let $(X, A)$ be a relative $C W$-complex, $Y$ a connected simple $C W$-complex, $g$ a map $A \rightarrow Y$, and $n$ a positive integer such that the $k$-invariant $k^{n+1}(Y)$ has finite order $s_{n}^{Y}$.

(a) If $g$ can be extended over $X_{n+1}$, then $\zeta^{n+1}(g)=0$.

(b) Assume that $g$ has an extension $\bar{g}: X_{n} \rightarrow Y$, that multiplication by $s_{n}^{Y}$ on $H^{n+1}\left(X, A ; \pi_{n} Y\right)$ is injective, and that $\zeta^{n+1}(g)=0$, then $g$ can be extended over $X_{n+1}$.

Proof. Assertion (a) is obvious since the extensibility of $g$ (and consequently of $f_{n}^{Y} \circ g$, for any $n$-dimensional characteristic map $f_{n}^{Y}$ ) over $X_{n+1}$ implies the vanishing of $\gamma^{n+1}\left(f_{n}^{Y} \circ g\right)=\zeta^{n+1}(g)$. In order to prove (b), we deduce from the hypothesis and Definition 3.2 that $s_{n}^{Y} c^{n+1}(\bar{g}) \sim 0$, and therefore that $e^{n+1}(\bar{g}) \sim 0$. It is then a consequence of $[5$, Extension theorem $I]$ that $\left.\bar{g}\right|_{X_{n-1}}$ can be extended over $X_{n+1}$.

Corollary 3.6. Let $(X, A)$ be a d-dimensional relative $C W$-complex, $Y$ an $(m-1)$-connected simple $C W$-complex $(m \geq 1)$, and $g$ a map $A \rightarrow Y$. Assume for $m+1 \leq n \leq d-1$ that $k^{n+1}(Y)$ has finite order $s_{n}^{Y}$ and that multiplication 
by $s_{n}^{Y}$ on $H^{n+1}\left(X, A ; \pi_{n} Y\right)$ is injective. Then $g$ can be extended over $X$ if and only if $\zeta^{n+1}(g)=0$ in $H^{n+1}\left(X, A ; \pi_{n} Y\right)$ for $m \leq n \leq d-1$.

Proof. Suppose that $\zeta^{n+1}(g)=0$ for $m \leq n \leq d-1$. Observe first that $\gamma^{m+1}(g)=(-1)^{m} \delta g^{*}\left(i^{m}(Y)\right)=0$ by Propositions 1.3 and $3.3:$ thus $g$ may be extended over $X_{m+1}$. We then apply inductively (for $n=m+1, m+2, \ldots, d-1$ ) assertion (b) of the previous theorem and obtain an extension of $g$ over $X_{d}=X$. The converse is trivial.

We consider finally the extension problem in the case where $Y$ is an $(m-1)$ connected infinite loop space : the obstruction classes $\zeta^{n+1}(g)$ may be defined because each $k$-invariant $k^{n+1}(Y)$ of the space $Y$ is a cohomology class of finite order $s_{n}^{Y}$ (note that any prime $p$ dividing $s_{n}^{Y}$ satisfies the inequality $p \leq$ $(n-m+3) / 2$ according to [3, Corollary 1.9]). For a positive integer $t$, let us call $M_{t}$ the product of all primes $p \leq t / 2+1$.

Corollary 3.7. Let $(X, A)$ be a d-dimensional relative $C W$-complex, $Y$ an $(m-1)$-connected infinite loop space $(m \geq 1)$, and $g$ a map $A \rightarrow$ $Y$. Let $R$ denote the ring $\mathbb{Z}\left[1 / M_{d-m}\right]$, $\ell$ the localization map $Y \rightarrow Y_{R}$, and $\ell_{*}: H^{n+1}\left(X, A ; \pi_{n} Y\right) \rightarrow H^{n+1}\left(X, A ; \pi_{n} Y \otimes R\right)$ the homomorphism induced by $\ell$. Then the composition $\ell \circ g: A \rightarrow Y_{R}$ is extensible over $X$ if and only if $\ell_{*}\left(\zeta^{n+1}(g)\right)=0$ in $H^{n+1}\left(X, A ; \pi_{n} Y \otimes R\right)$ for $m \leq n \leq d-1$. In particular, if $j^{n}(Y)$ is an $n$-dimensional characteristic class satisfying $\delta g^{*}\left(j^{n}(Y)\right)=0$ in $H^{n+1}\left(X, A ; \pi_{n} Y\right)$ for $m \leq n \leq d-1$, then $\ell \circ g$ has an extension $X \rightarrow Y_{R}$.

Proof. Since the map $\ell$ localizes the $k$-invariants, one has $s_{n}^{Y_{R}}=1$ for $n \leq d-1$. The previous corollary asserts that $\ell \circ g$ can be extended over $X$ if and only if $\zeta^{n+1}(\ell \circ g)=0$ in $H^{n+1}\left(X, A ; \pi_{n} Y \otimes R\right)$ for $m \leq n \leq d-1$; but $\zeta^{n+1}(\ell \circ g)$ vanishes if and only if $\ell_{*}\left(\zeta^{n+1}(g)\right)=s_{n}^{Y} \zeta^{n+1}(\ell \circ g)=0$. (cf. Remark $3.4(\mathrm{~b})$ ). Finally, if $\delta g^{*}\left(j^{n}(Y)\right)=0$, then $\ell_{*}\left(\zeta^{n+1}(g)\right)=(-1)^{n} \ell_{*}\left(\delta g^{*}\left(j^{n}(Y)\right)\right)=0$.

\section{References.}

1. Arkowitz, M., Curjel, C.R. : The Hurewicz homomorphism and finite homotopy invariants. Trans. Am. Math. Soc. 110, 538-551 (1964)

2. Arlettaz, D. : On the homology of the special linear group over a number field. Comment. Math. Helv. 61, 556-564 (1986) 
3. Arlettaz, D. : On the $k$-invariants of iterated loop spaces. Proc. R. Soc. Edinb. Sect. A 110, 343-350 (1988)

4. Borel, A. : Cohomologie réelle stable de groupes $S$-arithmétiques classiques. C.R. Acad. Sci. Paris Sér. A 274, 1700-1702 (1972)

5. Eilenberg, S. : Cohomology and continuous mappings. Ann. Math. 41, 231-251 (1940)

6. Peterson, F.P. : Some remarks on Chern classes. Ann. Math. 69, 414-420 (1959)

7. Sullivan, D. : Genetics of homotopy theory and the Adams conjecture. Ann. Math. 100, 1-79 (1974)

8. Whitehead, G.W. : Elements of Homotopy Theory. Graduate Texts in Math. 61. Berlin-Heidelberg-New York : Springer 1978

Dominique Arlettaz

Institut de mathématiques

Université de Lausanne

CH-1015 Lausanne

Switzerland 